Warum sind die besten Spitäler von Ärztinnen oder Ärzten geführt? Dies haben Sie sich sicher auch schon gefragt. Mehr darüber erfahren Sie von der Forscherin aus Zürich in diesem lesenswerten Interview.

\title{
«Evidenz spricht für Besetzung von Führungspositionen mit Ärzten»
}

\author{
Interview: Bruno Kesseli
}

Dr. med. et lic. phil., Chefredaktor

\author{
Sind Spitäler besser, wenn Sie von Ärztinnen und Ärzten geführt werden? Zu diesem \\ Thema forscht die an der Universität Zürich lehrende Ökonomin Agnes Bäker. Ihre \\ Erkenntnisse fasste sie im Mai in einem spannenden Referat vor der Ärztekammer \\ zusammen. Das folgende Interview knüpft an ihre damaligen Ausführungen an.
}

1 U.S. News \& World Report's rankings: Der U.S. News \& World Report ist ein amerikanisches Nachrichtenmagazin, das einflussreiche Rankings von Institutionen im Bildungs- und Gesundheitsbereich, aber auch auf weiteren Gebieten, publiziert.

\begin{abstract}
Frau Bäker, Sie haben mit zwei Mitautoren im vergangenen Jahr in der Harvard Business Review einen Artikel mit dem Titel «Why The Best Hospitals Are Managed by Doctors" publiziert. Wie kamen Sie dazu, sich mit diesem Thema zu beschäftigen?

Den Anstoss gab Amanda Goodall, eine Ko-Autorin des Artikels. Ich hatte Amanda zu einem Vortrag an meine damalige Universität eingeladen und sie stellte die Evidenz zu «Expert leadership» vor - unter anderem ihre 2011 veröffentlichte Studie zur Korrelation des Ranges von U.S. Spitälern im USNWR-Ranking ${ }^{1}$ mit dem ärztlichen Hintergrund des CEOs. Die Frage nach der Erklärung, den Mechanismen hinter dieser starken Korrelation beschäftigte mich sofort. Und seitdem versuchen wir dies - zunächst theoretisch, inzwischen auch empirisch - zu ergründen.
\end{abstract}

Die Titelaussage impliziert zunächst, dass die besten Spitäler von Ärztinnen oder Ärzten geführt werden. Aufgrund welcher Datenbasis lässt sich eine solche Aussage machen?

Diese Aussage, die ja zunächst nur eine Korrelation beinhaltet, lässt sich inzwischen an einer Reihe von Datensätzen zeigen. Amanda betrachtete die Top-100 U.S. Spitäler nach dem USNWR-Ranking von 2009. Eine eben veröffentlichte Studie von Tasi, Keswani und Bozic (2017) nutzt das USNWR-Ranking von 2015 und zeigt den Zusammenhang für die grössten 115 Spitäler

\section{Zur Person}

Prof. Dr. rer. pol. Agnes Bäker, geb. 1982, ist seit März 2016 Assistenzprofessorin (tenure track) für Management von Nonprofit-Organisationen an der Universität Zürich. Sie lehrt im CAS «Medical Leadership» des Instituts für Betriebswirtschaftslehre und im CAS

"Aortic Valve Structural Interventions" des Universitätsspitals Zürich - zwei Programme, die sich speziell an Ärztinnen und Ärzte wenden und unter anderem der Vermittlung von Managementwissen und Führungsfähigkeiten widmen.

in den USA, von denen 34 von Ärztinnen oder Ärzten geleitet wurden. Allerdings finden die Autoren keine Unterschiede in ökonomischen Kennzahlen, nur in Qualitätsmassen für die Spitalleistung. Eine Studie von Kuntz und Scholtes aus dem Jahr 2013 ergibt, dass die Anzahl der Ärztinnen und Ärzte pro Patient und der Pflegekräfte pro Patient grösser ist in Krankenhäusern mit einem ärztlichen CEO in Vollzeit als in Teilzeit. Für Spitäler in England können Veronesi, Kirkpatrick und Vallascas (2013) zeigen, dass die Leistung der Spitäler grösser ist, wenn der Anteil der Ärztinnen und Ärzte im Aufsichtsrat grösser ist. In einem Überblicksartikel fassen Sarto und Veronesi (2016) zusammen, dass die Evidenz für die Besetzung von Führungspositionen in Spitälern mit Ärzten spricht. 


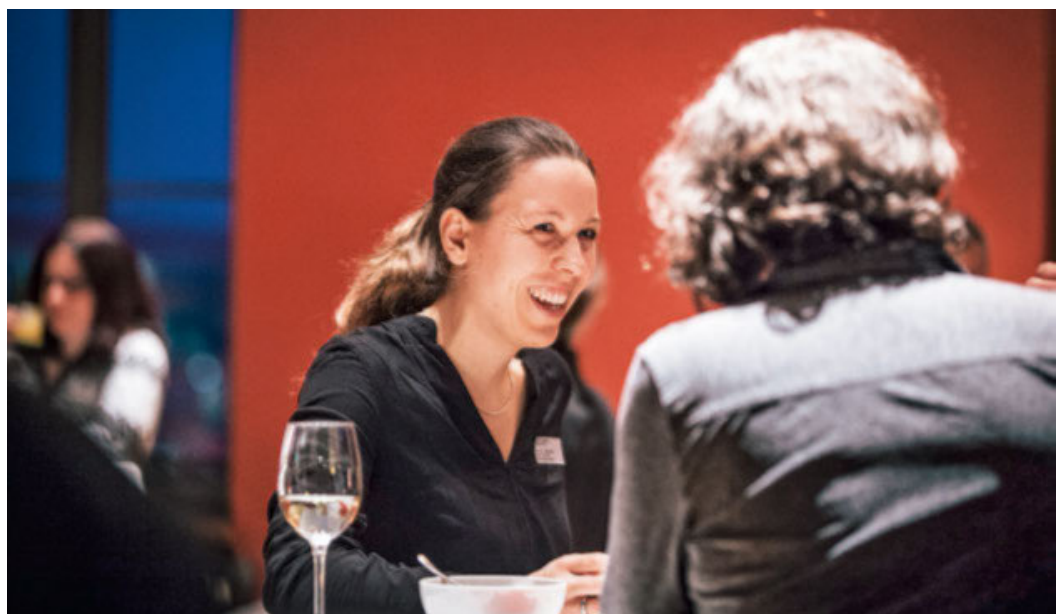

Agnes Bäker forscht und publiziert zum Thema, wie Ärztinnen und Ärzte im TopManagement sich auf die Performance von Spitälern auswirken.

Wenn von «den besten Spitälern» die Rede ist: An welchen Kriterien werden diese gemessen? Das ist je nach Studie unterschiedlich. Die Studien von Amanda und von Tasi und Koautoren messen Spitalqualität über das USNWR-Ranking. Tasi und Koautoren ziehen ausserdem noch die Bettenbelegung heran. Für den Gewinn und die Gewinnspanne finden sie hingegen keine signifikanten Unterschiede. Die Studie von Kuntz und Scholtes betrachtet das Verhältnis aus Ärzten und Patienten. Gerade die Tatsache, dass der Befund anscheinend nicht von der Wahl des Masses für die Spitalleistung abhängt, macht diesen für mich umso stärker. Gleichzeitig muss man auch sehen, dass nicht in allen Studien für alle Leistungsmasse ein statistisch signifikanter Unterschied gefunden wird.

"An Zufall glaube ich in diesem Zusammenhang nicht, dafür sind die Befunde zu replizierbar.»

Der Zusammenhang zwischen Ärzten an der Spitze und besserer Spitalperformance lässt sich offenbar statistisch nachweisen. Gibt es Indizien für einen kausalen Zusammenhang oder könnte es sich genauso gut um eine zufällige Assoziation handeln? An Zufall glaube ich in diesem Zusammenhang nicht, dafür sind die Befunde zu replizierbar. Um Kausalität nachzuweisen, müsste man jedoch ein Experiment durchführen, in dem man Spitälern randomisiert ärztliche und nicht-ärztliche Führungskräfte zuweist. Und auch nach langem Suchen haben wir bisher kein Spital gefunden, das damit einverstanden wäre. Was bleibt sind Langzeitstudien, in denen die Spitalleistung über die Zeit hinweg analysiert wird und mit etwaigen Wechseln an der Spitze in Verbindung gebracht wird.
Für Spitäler haben wir diese Studien noch nicht durchgeführt. Amanda hat jedoch unterstützende Evidenz auf einem verwandten Gebiet gefunden: Die Ernennung häufig zitierter Forscher zum Fachbereichssprecher an Universitäten hat einen positiven Effekt auf die langfristige Forschungsleistung des Fachbereichs.

Gibt es Erkenntnisse darüber, wie sich Management by doctors in den verschiedenen Bereichen eines Spitals auswirkt? Welche Mechanismen sind dafür verantwortlich, dass es den Patienten am Schluss besser geht?

Genau die Frage nach den Mechanismen finde ich besonders spannend. Auf mittlerer Führungsebene in Spitälern sehen wir in unserer jüngsten Studie, dass klinische Expertise mit einem anderen Führungsstil und anderen Human-Ressources-Praktiken einhergeht. Zudem wird das Arbeitsumfeld von den unterstellten Ärztinnen und Ärzten positiver wahrgenommen. Mög licherweise sind es also diese Praktiken des Personalmanagements, die den Ausschlag geben. Allerdings denken wir, dass für die Top-Management-Ebene die

«Was wir zeigen können, ist, dass Ärzte mit klinisch exzellenten Führungskräften zufriedener mit den Führungspraktiken und dem Arbeitsumfeld sind.»

höhere Glaubwürdigkeit des CEOs, wenn diese(r) eine exzellente Ärztin oder ein exzellenter Arzt ist, eine zentrale Bedeutung hat. Dies könnte zum Beispiel dazu führen, dass Entscheidungen weniger angezweifelt werden. Bisher haben wir dafür jedoch nur anekdotische Evidenz.

\section{Wie sieht die wirtschaftliche Performance von Spitälern aus, die von Ärztinnen oder Ärzten geleitet werden?}

Zumindest die Studie von Tasi, Keswani und Bozic (2017) findet keinen Unterschied - was ja letztendlich ein guter Befund ist, bedeutet es doch, dass finanzielle Erwägungen nicht im Widerspruch zu einer optimalen Patientenversorgung stehen.

Kommen wir zurück zur Titelfrage Ihres Artikels: Worauf ist es zurückzuführen, dass von Ärztinnen und Ärzte geführte Spitäler besser sind als solche ohne Ärzte an des Spitze des Managements?

Abschliessend können wir das (noch) nicht beantworten, da sich nicht alle unserer theoretischen Mechanismen gut erfassen lassen. Beispielsweise ist es schwer, die Glaubwürdigkeit der Führungskraft zu messen oder den genauen Zusammenhang zwischen der klini- 
schen Expertise einer Führungskraft und der Qualität der neueingestellten Ärzte zu ergründen. Homophilie könnte dazu führen, dass klinische Experten andere klinische Experten einstellen - aber für diese Analyse bräuchte man Informationen über die Qualität aller Bewerber beziehungsweise Kandidaten. Was wir zeigen können, ist, dass Ärzte mit klinisch exzellenten Führungskräften zufriedener mit den Führungspraktiken und dem Arbeitsumfeld sind. Sie fühlen sich zum Beispiel sicherer vor Mobbing oder Diskriminierung. Über das Arbeitsumfeld wird dann auch die Leistung der Ärztinnen und Ärzte beeinflusst, und damit die Spitalleistung. Was uns somit noch fehlt, ist die Verbindung zwischen den Ärzten an der Spitze und den klinischen Experten auf mittlerer Führungsebene. Hier gehen wir davon aus, dass Praktiken von oben nach unten weitergegeben werden und auch eine gewisse Selektion und Passung vorliegt, da obere Hierarchieebenen für die Besetzung von unterstellten Positionen verantwortlich sind.

"Von daher würde ich sagen: Ja, die Erkenntnis, dass Experten im Kerngeschäft ceteris paribus die besseren Führungskräfte sind, gilt allgemein.»

\section{Sind die im Artikel vorgestellten Erkenntnisse zur Expert Leadership ärztespezifisch oder lassen sie sich verallgemeinern?}

Bei der Verallgemeinerung auf andere Kontexte muss man natürlich immer vorsichtig sein. Allerdings gibt es inzwischen unterstützende Evidenz für Expert Leadership aus einer Reihe von anderen Kontexten wie Universitäten, Basketball, Formel 1 und sogar branchenübergreifende Befunde aus grossangelegten Arbeitnehmerbefragungen. Von daher würde ich sagen: Ja, die Erkenntnis, dass Experten im Kerngeschäft ceteris paribus die besseren Führungskräfte sind, gilt allgemein. Was die Mechanismen anbelangt, so finden wir vergleichbare Befunde auch in Universitäten, was immerhin ein Zeichen für die Verallgemeinerbarkeit ist.

Welche weiteren interessanten Befunde im Zusammenhang mit der untersuchten Fragestellung würden Sie hervorheben?

Ich denke es ist wichtig zu erwähnen, dass natürlich nicht jeder Arzt oder jede Ärztin eine exzellente Führungskraft ist. Management- und Führungsfähigkeiten werden entsprechend in unserem theoretischen Modell berücksichtigt, aber erst mit unserer jüngsten Befragung von Klinikern können wir nun tatsächlich auch zeigen, dass die administrative Expertise der ärztlichen Führungskräfte eine entscheidende Rolle für die Arbeitszufriedenheit spielt.

Aus Ihren Ergebnissen liesse sich der Schluss ziehen, dass Spitäler Ärztinnen und Ärzte als CEOs anstellen müssen, um erfolgreicher zu werden. Ist eine solche Schlussfolgerung zulässig?

Amanda und ihre Koautoren zeigen, dass sich die Forschungsleistung von universitären Fachbereichen verbessert, wenn man einen vielzitierten Forscher anstellt. Verallgemeinert man diesen Befund, dann könnte es auch für Spitäler sinnvoll sein, ärztliche CEOs einzustellen. Aber bisher haben wir, wie beschrieben, keine auch nur annähernd kausale Evidenz für Spitäler. Ausserdem stört mich an der Aussage, dass sie impliziert, nur wenn man einen ärztlichen CEO einstellte, könne die Spitalleistung besser werden. Dem ist natürlich nicht so.

\section{Lassen sich aus Ihren Erkenntnissen weitere Empfeh-} lungen für die Praxis ableiten?

Dass es wichtig ist, Ärztinnen und Ärzte für Führungsaufgaben zu schulen und nicht nur für die klinische Tätigkeit. Gleichzeitig wird man auch das Thema angehen müssen, dass Ärztinnen und Ärzte nicht um ihre Identität als Mediziner/-in fürchten müssen, wenn sie ihre Führungstätigkeit ernst nehmen und Führungspositionen - bis zur Position des CEOs - übernehmen.

Welche Fragen zum behandelten Themenkreis sollten in weiteren Studien vertieft untersucht werden? Haben Sie selbst diesbezügliche Projekte?

"Ich denke es ist wichtig zu erwähnen, dass natürlich nicht jeder Arzt oder jede Ärztin eine exzellente Führungskraft ist.»

Meine beiden Koautoren und ich sind in verschiedene angewandte Projekte involviert, im Rahmen derer wir Management- und Leadership-Programme für Ärztinnen und Ärzte aufsetzen - und diese auch wissenschaftlich evaluieren. Gleichzeitig haben wir mehrere Forschungsprojekte, die sich dem «Why» in unserem Artikel näher widmen. Und auch das Thema der Identität als Arzt oder Manager und wie man Ärztinnen und Ärzte davon überzeugen kann, CEO-Positionen zu übernehmen, wird in einem zukünftigen Projekt untersucht werden.

\section{Bildnachweis:}

Porträtfoto Universität Zürich.

Foto Agnes Bäker im Gespräch: @ Sabine Biedermann. 